\title{
Developing mHealth Apps with Researchers: Multi-Stakeholder Design Considerations
}

\author{
Michael P. Craven ${ }^{1,3}$, Alexandra R. Lang ${ }^{2}$, and Jennifer L. Martin ${ }^{3}$ \\ ${ }^{1}$ The University of Nottingham, Electrical Systems \& Optics Research Division, \\ Faculty of Engineering, University Park, Nottingham NG7 2RD, United Kingdom \\ michael.craven@nottingham.ac.uk \\ ${ }^{2}$ The University of Nottingham, Human Factors Research Group, \\ University of Nottingham, University Park, Nottingham NG7 2RD, United Kingdom \\ alexandra. lang@nottingham.ac.uk \\ ${ }^{3}$ NIHR MindTech Healthcare Technology Co-operative, The Institute of Mental Health, \\ Jubilee Campus, Nottingham, NG7 2TU, United Kingdom \\ \{michael.craven, jennifer.martin\}@nottingham.ac.uk
}

\begin{abstract}
The authors have been involved with developing a number of mHealth smartphone Apps for use in health or wellness research in collaboration with researchers, clinicians and patient groups for clinical areas including Sickle Cell Disease, Attention Deficit Hyperactivity Disorder, asthma and infertility treatment. In these types of applications, end-users self-report their symptoms and quality of life or conduct psychometric tests. Physiological data may also be captured using sensors that are internal or external to the device. Following a discussion of the multiple stakeholders that are typically involved in small scale research projects involving end-user data collection, four Apps are used as case studies to explore the issue of non-functional requirements.
\end{abstract}

Keywords: m-Health, Requirements Engineering, Software Engineering, User experience, Ethical issues in DUXU, Healthcare/Medical systems and DUXU, Management of DUXU processes, Medical/healthcare and DUXU, Mobile products and services.

\section{Background}

Mobile health (mHealth) applications (Apps) based on cellular phones, Smartphones and tablet computers are a rapidly growing trend in healthcare [1-3]. Healthcare researchers are increasingly turning to mobile apps for data collection as it is seen as a quick, easy way to obtain data from end-users (patients, carers or members of the public) in their everyday environment, using devices that users either own themselves or are becoming increasingly familiar with.

Apps can be used for a variety of purposes for monitoring or self-reporting of a person's health state or well-being (including performing tests) and to assist with tracking of adherence to medication use and/or treatment. They may also capture 
and/or process data sources from sensors that are capable of physiological measurement that are either internal to the device (accelerometer, gyroscope, camera, microphone, GPS) or external where they are carried or are worn on (or potentially in) the body or clothes or are present in the near environment [4].

However, it has been noted that Apps are often designed with little health professional involvement [5,6] and that end-user Apps are often produced from the healthcare system perspective rather than with a user-centred approach [7]. In addition, the importance of a number of other stakeholder considerations are evident, including ethical and other research governance requirements and potential for interaction with healthcare informatics infrastructure within the research institution or provider organisation(s). Some of these themes are common to other healthcare technologies or software, whereas others are the result of the mobile nature of the devices and the relatively uncontrolled environments in which they operate.

\section{Multiple Stakeholders}

A typical set of stakeholders for an App being developed for use in a small research project is shown in Fig. 1. This consists of four main groups: research institution and/or healthcare provider organisation, researchers, users and applications developers.

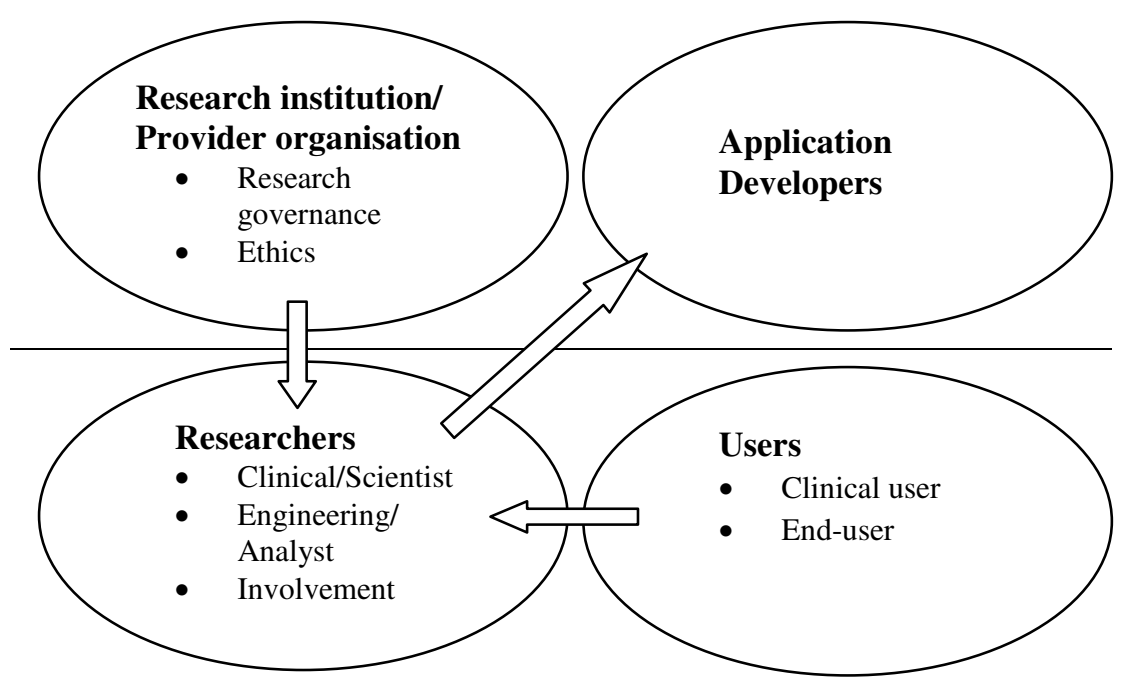

Fig. 1. Stakeholder groups in the App design process and flow of requirements

The make-up of an App development project team for a research application may include some or all of clinical/scientist, engineering/analyst and involvement researchers who will all have a role in software requirements production and also have their own requirements, not least because there is usually a tight timeline for completing the study. 
In a university, for example, a research collaboration could include all three types of researcher. The goals for the researchers will be the effective testing of an App prototype for its functionality, usability and reliability in a real-world setting and to then use it to successfully capture end-user data for the study.

The clinical/scientist researcher will typically supply the App's requirements in terms of clinical or wellness outcomes (from prior knowledge or from liaison with providers) and will usually provide the study design methodology to be implemented e.g. questionnaire or psychometric test. A clinical/scientist researcher may also be a user especially at the early prototype stage or in a small study. An involvement researcher will ensure clinical user or end-user engagement throughout the process. The engineering researcher, if any, acting as a software analyst, will be responsible for producing the Apps requirements for the application developer or alternatively they may also be involved in the application development and implement the requirements themselves.

In software engineering the 'how?' of implementation is generally separated from the requirements process. However, there are some aspects of mobile development which mean that non-functional requirements $[8,9]$ may strongly influence the choice of implementation platform and so for the application developer a number of implementation choices have to be made in parallel with the requirements process [10] e.g. What range of devices and operating systems should be supported? What device functions will be available and which are likely to be used by other applications? For mobile Apps this quickly leads to the question of whether to choose a native, web or hybrid implementation [11]. Furthermore, unless the App is standalone there is a question of server-side interfacing and interoperability across healthcare IT systems which may be prescribed or regulated though an API or interoperability toolkit. The use of Apps and sensors in a network may demand that a range of security issues be addressed in the system design [12].

Additional non-functional issues to be addressed in the development process include fully eliciting end-user \& clinical/scientist user needs and fulfilling ethical and research governance requirements of the research institution or healthcare provider organisation(s). It is likely these will be addressed via the research team by liaison with clinical or end-users and with appropriate review of the proposed research by institution or provider contacts.

Ethical requirements include the practical aspects of ensuring personal data security and anonymity. Other ethical factors are privacy during the study (in particular when the App is dealing with sensitive information), burden on the patient relating to the degree of active or passive monitoring and the frequency of data collection, and impact on clinical care (e.g., potential impact on health in the course of App use due to possible stress or anxiety). Assessment of such ethical issues at an early stage of technology development is advisable. Trust in such security and privacy measures by the users and confidence in the system in general should help them offer honest and full responses, whilst early consideration of user requirements and interactions will improve the design of the research study through an improved understanding of burden, potentially leading to reduced withdrawal/drop-out rates.

User requirements include identifying all potential users and their capabilities, needs and preferences. As with Apps in general, users' physical and cognitive 
abilities, prior experience of using computer systems and the internet and preferences for modes of communication will all have an impact in mHealth. Also relevant are practical issues when devices are used for everyday interaction at the same time as being used for monitoring health, including text messaging and use of native phone functions such as accelerometers, alarms, microphones and cameras. Participants in research studies may rely on existing usage of these phone functions and so the mixing of their normal daily use and use for a study may conflict. We have proposed audits with the clinical population as a way of exposing these requirements $[4,11]$ in addition to more traditional methods such as focus groups or drop-in sessions with early prototypes.

Furthermore, mHealth App users do not always keep devices turned on or charged up. For a research study this could be inconvenient or result in loss of data. However, a research instrument may also become a product and if an App is being used to monitor care it will need to be rigourously assessed for accuracy and reliability.

Constant and honest communication about requirements and expectations between researchers and App developers is essential to prevent frustration, misunderstandings, or problems when the research starts. For example, if the end-user is frustrated or has a poor experience then this may result in poor adherence or withdrawal from the study. Researchers need to be able to tell the developers what they require and in turn clinical researchers need to know the capabilities (and restrictions) of the technology so that their expectations are realistic. Developers on the other hand, will need to know about potential ethical restrictions at the outset.

This paper now draws out some of the stakeholder requirements from four case studies of App developments with researchers, all of which include one or more of the authors as the researcher(s).

\section{Case Studies}

\subsection{Mild Asthma Self-reporting with and without Physiological Measurement}

The first case study concerned persons with mild asthma. This project (described in more detail in a previous paper [4]) was a pilot study of self-reporting by means of a daily smartphone questionnaire without and then with additional twice-daily physiological measurements from a pulse oximeter and peak flow meter, to study user requirements and interactions between self-reporting and the measurement tasks.

The stakeholders in this study were University of Nottingham researchers in the Faculty of Engineering and School of Computer Science who were conducting the user requirements study and the end-users were recruited from volunteers at the university identifying themselves as having mild asthma. The application development was carried out by a graduate student.

From the researchers there was a requirement for a quick solution since the study was due to take place over 12 weeks. Since one component was a text-based questionnaire (requiring readings from peak flow meter to be entered into the questionnaire manually), it was decided to implement the study as a web App and use an inexpensive third party Android App to capture data from a Bluetooth pulse 
oximeter. where Android smartphones would be lent to the users by the project researchers over a period of 2 weeks, therefore it was decided to implement the study on an Android platform only.

The other main non-functional requirement prior to the study commencing was ethical. The ethics committee expressed a requirement for data security which was fulfilled by using an HTTPS connection with password protection and for analysis the data was downloaded over a secure connection to university computers, with the usual safeguards restricting access to named personnel. No user identification was collected or stored on the phone. In addition, participants were able to set a passcode to lock the phone, preventing unauthorised access during use.

From the end-user perspective a number of non-functional requirements were identified during the pilot study including convenience (e.g. inconvenience from sitting down to use the pulse oximeter), confidence in the success of data upload and dependability of the Wi-Fi, Bluetooth and battery. Another requirement was that the App should not affect the users' condition and which was not evident during the study although one participant did express the opinion that thinking about a cough may exacerbate it. On a positive note, one participant reported that using the App acted as a reminder to carry their asthma inhaler.

\subsection{IVF Treatment Stress Diary}

The second project concerned women undergoing in-vitro fertilisation (IVF) treatment [11]. The requirement was for ecological momentary assessment during IVF treatment using patients' own phones, allowing them to complete entries in a stress diary and respond to prompts to perform a psychological test (IPANAT [13]) in a secure manner.

The requirements were supplied by a postgraduate psychology student and their research supervisors at The University of Nottingham, School of Medicine one of whom was a Consultant Gynaecologist. They also organised patient recruitment. The requirements were produced by researchers in the Faculty of Engineering and application development was carried out by an in-house application programmer who was also a researcher on the project.

Since patients were to use their own phones, a phone survey was first carried out in which all participants were found to have access to some kind of mobile device and three-quarters of these were found to own a smartphone and use Apps [10]. Communication preferences showed a majority preference for an App to collect diary and test results and communicate them to the researchers but some participants' preferences were for other modes of communication (SMS text, voice, paper questionnaire). Furthermore, wide usage of an alarm clock function was found.

The subsequent ethics committee submission to an NHS panel for the main study raised some interesting non-functional requirements. In particular, the panel thought that text messaging for prompting could potentially compromise confidentiality and security of the data if the phone was lost. The advantage of using an App would be that data would be recorded on a secure server with password protection. Also for this particular project, the potential use of telephone conversations as a method of prompting/signalling users to carry out the ecological assessment was considered to 
be an unacceptable burden because this would entail contacting the patients every two days. This would also have been a burden on the researcher.

Due to the requirement for patients to use their own phones, and the fact that the survey found that most participants had either an Android phone or iPhone, it was decided to implement an App for each type of phone. The native alarm would be more acceptable for prompting since this could be turned off by the user and did not reveal the source of the prompt. Implementation of the App in Android phones used Eclipse Integrated Development Environment (IDE) with the Android Development Tools plug-in and for iPhones, Xcode was used [11].

\subsection{Sickle Cell Disease Pain App}

The aim of this project was to develop a valid, reliable and acceptable method of remotely monitoring blood oxygen saturation $\left(\mathrm{SpO}_{2}\right)$ for people with Sickle Cell Disease (SCD). Two data collection studies were planned. The first was a focus group study to collect subjective information from adults with SCD. The results of this were used to inform the design of the second study: a feasibility trial of a new device for home monitoring of $\mathrm{SpO}_{2}$ using a Bluetooth pulse oximeter (using the same device as in case study 3.1) interfaced to a custom designed App (instead of the third party App in 3.1).

Stakeholders included researchers at the University of Nottingham, Faculty of Engineering including the same in-house developer as in 3.2 and the Sickle Cell Society who assisted with the recruitment of volunteers with SCD.

The aim of the feasibility trial was to test the function of the sensors to monitor variations in oxygen saturation and to gauge the acceptability of the monitoring process to the participants with SCD. Participants were given Android phones and a pulse oximeter for a period of 3 months. During this time they were be asked to take their $\mathrm{SpO}_{2}$ readings twice a day and to record symptoms of pain and answer questions about quality of life and breathlessness several times each day, according to their location. No clinical decisions about treatment were to be made on the basis of the readings.

The requirement to include questions about symptoms of SCD in the App came from both the researcher and the users, however, this was for different reasons. Individual users wished to be able to report exactly how they were feeling in a way that was natural and meaningful to them and this varied somewhat from person to person. For the researcher, however, it was important that symptom information was collected in a standardised way that would allow comparisons to be made between users and across time.

This was resolved by a number of informal and group discussions between the researcher and potential users which aimed to discover how they talked about their symptoms with clinicians and how they talked about them with their families. The result was that validated clinical scales were used that met the requirements of the researcher but additional questions were added based on the views of the users. For example, rather than just asking about general pain, users were able to record the body area that they were experiencing pain.

Many people within the user population for this study (adults with SCD) come from disadvantaged backgrounds and a large number of potential users did not own a 
smartphone. As a result phones were lent to users for the duration of the study and because of budget constraints the decision was made to develop an Android app because of the lower cost of Android handsets.

Implementation of the App was in Eclipse IDE with the Android Development Tools plug-in.

\subsection{Snappy App: Attention Deficit Hyperactivity Disorder Assessment App}

Attention Deficit Hyperactivity Disorder (ADHD) is a neurodevelopmental syndrome that is characterised by three core symptom domains; inattention, hyperactivity and impulsivity. Given the variation in causes and behavioural consequences of ADHD, diagnosis, symptom monitoring and response to medication currently rely on subjective interpretation of information gained through clinical interview and questionnaires. Standardised measures of cognitive function offer the potential to provide a more objective measure of symptoms and response to medication, and could potentially speed up the process of treatment optimisation.

A pilot study was conducted on a non-clinical sample to establish the feasibility and validity of a smartphone application which could be appropriate for monitoring symptoms in a clinical population. The researcher preference was for users to use their own phones because of budget constraints.

The stakeholders supplying the requirements are psychology researchers in the School of Medicine and researchers in the School of Engineering who produced the requirements and these were initially implemented as a smartphone App by the same in-house application developer as for 3.2 and 3.3.

The primary aim of the pilot study is to establish whether it is feasible for endusers to conduct a continuous performance test (CPT) [14] whilst their physical activity is measured using the in-built motion sensors (accelerometer, gyroscope) [15]. This type of psychometric test involves presenting a sequence of letters (or alternatively a series of images or audio cues) and asking the user to respond when a specified target occurs e.g. by clicking a switch, or in this case via the touch screen or a button on the screen of the smartphone, whilst remaining passive to non-target stimuli.

Initially, due to time constraints, an App was developed to implement the CPT on an Android platform only. Development in Eclipse with the Android Development Tools plug-in employed a Java API to access the accelerometer and (where present) gyroscope readings, during presentations of the CPT cues. The native alarm function was used to prompt the users to take the test. However, since the test was to be conducted at a low frequency of twice a week and there had been some problems with the alarm setting function in initial tests with users, it was subsequently deemed not too burdensome to email the participants instead. At the same time the researchers also expressed a preference to widen the platform to iPhones but it was no longer possible to use the original in-house application developer.

As a result, with an extra month to make the change, it was decided to switch the implementation to a web App written in Javascript using HTML5 device motion and orientation features to access the accelerometer and gyroscope data and to dispense with the native alarm function which would not be controllable from a web client. 
Server collection of the data was implemented using HTTPS. The web App was implemented by another of the Engineering researchers.

For the researchers, meaningful correlations were found in this initial study with activity data collected from the phone sensors however it was found that it was possible to collect accelerometer data from only 6 of 11 phones and just 4 phones were able to provide gyroscope orientation data [15]. This situation may improve in the future when more phones support HTML5 functions. Results from the study from the end-user point of view showed that the task was considered to be easy and not stressful, however preferences were expressed about screen background colour, font size, display of the time, and a number of usability suggestions included choice of user name, addition of a help function, further reminders (by text messaging) and a gamification function (scoring or ranking system for participants).

\section{Conclusions}

The results of the four case studies give some insight into the production of researchled Apps which were produced, implicitly, through a Scrum-like process [10] using a small in-house development team.

Non-functional requirements were dominated by development time from the researcher point of view and are seen to influence the implementation choice e.g. limiting it to one platform. Cost requirements also acted to limit implementation choices. Data security, privacy and concern for end-user burden dominated the requirements of the research institution ethics committees, which in turn limited implementation choices, in one case excluding text-messaging in favour of the native alarm clock for prompting the end-user (case study 3.2). Ethical challenges associated with App-based research are an ongoing issue for which theory and regulation is still catching up with current opportunities arising from the use of these technologies for research purposes.

From user feedback in case study 3.1 a number of user requirements were revealed including reliability of data collection and dependability of device networking and power. In 3.3 the App was required by end-users to produce information beyond that required by the researchers and in 3.4 there were several aesthetic and usability requirements that emerged, some of which may conflict with ethical or researcher requirements.

Device-independence is an implicit requirement that is mostly not being met and choices were made in each of the examples which acted to exclude some devices and hence also either excluded their users, or demanded that they use phones that were lent to them by the researchers. An interesting solution is a modular framework for cross-platform development, LambdaNative, devised by Petersen et al. for mHealth applications, using the Scheme (Gambit $\mathrm{C}$ ) language, where they describe an oximeter application, a wireless monitoring and messaging device for multi-bed patient data and an anesthesia drug controller implemented as a client-server system. However, they accept that the use of Scheme will present a learning curve for engineering and the medical community personnel and that this had been the biggest challenge in their work so far (their development team consisted of four code developers on staff; one programmer, two physicists and one engineer, with diverse coding backgrounds) [16]. 
Requirement for the collection of sensor data from devices provides an additional challenge where it is seen from the result of 3.4 where not all phones provided motion and/or orientation data, in part due to variable support for HTML5 Javascript functions in the web App.

Due to the nature of the case studies we have not been able to explore interoperability or other informatics issues but this will involve further requirements production, in particular from provider organisation stakeholders.

Acknowledgements. The research reported in this paper was conducted by the National Institute for Health Research MindTech Healthcare Technology Cooperative (NIHR MindTech HTC) and funded by the NIHR. The views expressed are those of the author(s) and not necessarily those of the NHS, the NIHR or the Department of Health. All of the authors acknowledge additional support for this work through the Multidisciplinary Assessment of Technology for Healthcare (MATCH) programme (EPSRC Grant EP/F063822/1). MC and JM acknowledge support from MindTech. MC was involved with the asthma, IVF and ADHD (SnappyApp) projects, JM with the Sickle Cell project. The ADHD study was supported by MindTech and MATCH. The asthma diary project was supported by MATCH and a Xerox Research Centre Europe (Grenoble) donation for the support of studentships in Ubiquitous Computing within the Mixed Reality Laboratory at the University of Nottingham. The IVF project was supported by MATCH and Nurture Fertility. JM wishes to thank the Sickle Cell Society for its involvement in user testing. The authors wish to thank the other collaborators who are mentioned in the citations associated with the case studies.

\section{References}

1. World Health Organization: mHealth: New horizons for health through mobile technologies: second global survey on eHealth (2011), http: / /www . who. int/goe/ publications/goe_mhealth_web.pdf (accessed January 28, 2014)

2. Free, C., Phillips, G., Galli, L., Watson, L., Felix, L., Edwards, P., Patel, V., Haines, A.: The effectiveness of mobile-health technology-based health behaviour change or disease management interventions for health care consumers: a systematic review. PLoS Medicine 10(1), e1001362 (2013)

3. Mosa, A.S.M.M., Yoo, I., Sheets, L.: A Systematic Review of Healthcare Applications for Smartphones. BMC Medical Informatics and Decision Making 12, 67 (2012)

4. Craven, M.P., Selvarajah, K., Miles, R., Schnädelbach, H., Massey, A., Vedhara, K., Raine-Fenning, N., Crowe, J.: User requirements for the development of Smartphone selfreporting applications in healthcare. In: Kurosu, M. (ed.) HCII/HCI 2013, Part II. LNCS, vol. 8005, pp. 36-45. Springer, Heidelberg (2013)

5. Rosser, B.A., Eccleston, C.: Smartphone applications for pain management. Journal of Telemedicine and Telecare 17(6), 307-312 (2011)

6. Buijink, A.W.G., Visser, B.J., Marshall, L.: Medical apps for smartphones: lack of evidence undermines quality and safety. Evidence Based Medicine 18(3), 90-92 (2013) 
7. McCurdie, T., Taneva, S., Casselman, M., Yeung, M., McDaniel, C., Ho, W., Cafazzo, J.: mHealth Consumer Apps: The Case for User-Centered Design, AAMI Horizons, 49-56 (Fall 2012)

8. Chung, L., do Prado Leite, J.C.S.: On Non-Functional Requirements in Software Engineering. In: Borgida, A.T., Chaudhri, V.K., Giorgini, P., Yu, E.S. (eds.) Mylopoulos Festschrift. LNCS, vol. 5600, pp. 363-379. Springer, Heidelberg (2009)

9. Miller, R.E.: The Quest for Software Requirements. Maven Mark Books, Milwaukee (2009)

10. Wassermann, A.I.: Software Engineering Issues for Mobile Application Development. In: Proc. FSE/SDP Workshop on Future of Software Engineering Research (FoSER 2010), Santa Fe, New Mexico, USA, November 7-8, pp. 397-400. ACM, New York (2010)

11. Selvarajah, K., Craven, M.P., Massey, A., Crowe, J., Vedhara, K., Raine-Fenning, N.: Native Apps versus Web Apps: Which Is Best for Healthcare Applications? In: Kurosu, M. (ed.) HCII/HCI 2013, Part II. LNCS, vol. 8005, pp. 189-196. Springer, Heidelberg (2013)

12. Kumar, P., Lee, H.-J.: Security Issues in Healthcare Applications Using Wireless Medical Sensor Networks: A Survey. Sensors 12, 55-91 (2012)

13. Quirin, M., Kazén, M., Kuhl, J.: When nonsense sounds happy or helpless: The Implicit Positive and Negative Affect Test (IPANAT). J. Pers. Soc. Psychol. 97(3), 500-516 (2009)

14. van Leeuwen, T.H., Steinhausen, H.C., Overtoom, C.C., Pascual-Marqui, R.D., van't Klooster, B., Rothenberger, A., Sergeant, J.A., Brandeis, D.: The continuous performance test revisited with neuroelectric mapping: Impaired orienting in children with attention deficits. Behavioural Brain Research 94, 97-110 (1998)

15. Young, Z., Craven, M.P., Groom, M., Crowe, J.: Snappy App: a mobile continuous performance test with physical activity measurement for assessing Attention Deficit Hyperactivity Disorder. In: Kurosu, M. (ed.) Human-Computer Interaction, Part III, HCII 2014. LNCS, vol. 8512, pp. 363-373. Springer, Heidelberg (2014)

16. Petersen, C.L., Gorges, M., Dunsmuir, D., Ansermino, J.M., Dumont, G.A.: Functional Programming of mHealth Applications. In: Proc. International Conference on Functional Programming (ICFP 2013), Boston, USA, June 25-27, pp. 357-362. ACM, New York (2013) 